\title{
JEDI: AVALIAÇÃO PRELIMINAR DE UM PROGRAMA DE INCLUSÃO DIGITAL E SOCIAL
}

\author{
Gislane Santana ${ }^{1}$, Luiza Alonso ${ }^{1}$, Edilson Ferneda ${ }^{1}$, Daniel deOliveira ${ }^{3}$ \\ 1 'Programa de Mestrado em Gestão do Conhecimento e Tecnologia da Informação da \\ Universidade Católica de Brasília \\ ${ }^{3}$ Brasília Java Users Group - DFJUG \\ santana1204@gmail.com, lualonso@pos.ucb.br, eferneda@pos.ucb.br, dani- \\ el@dfjug.org
}

Resumo. O tema Inclusão Social e Digital tem sido objeto de debates no meio acadêmico, no mundo empresarial e no governo. As ações de projetos nessa área têm sido divulgadas, discutidas e recebidas por toda a sociedade. Entretanto, após a participação nesses projetos, não se conhece o destino dos que participaram de programas de inclusão social e digital. Pode-se afirmar que se tornaram digitalmente incluídos, mas, da mesma forma, pode-se dizer que se tornaram socialmente incluídos? Esses projetos de fato atingiram seus objetivos iniciais quanto aos objetivos propostos? Esse artigo aborda uma forma de se avaliar o impacto de uma iniciativa de inclusão digital no individuo e no mercado de trabalho a partir de um estudo de caso.

Palavras-chave: Terceiro setor. Inclusão digital. Gestão do conhecimento. Redes sociais. Avaliação.

\section{JEDI: PRELIMINARY ASSESSMENT OF A PROGRAM AND SOCIAL INCLUSION}

\begin{abstract}
Digital and Social Inclusion has been object of debates in the academic environment, in the business world and in the government. The actions of projects in that area has been divulgated, discussed and received by all the society. However, after participation in those projects, one does not know the fate of those who had participated on digital and social inclusion/enclosure projects. It is possible to affirm that they became digital included, but, similarly, it is able to be said that they became socially included? These projects actually achieved their initial goals as it was proposed? Using a case study, this article discusses a specific proposal for impact evaluation of a digital inclusion initiative at the individual and the job market level.
\end{abstract}

Keywords: Third Sector. Digital inclusion. Knownledge management. Social networks. Evaluation.

\section{Introdução}

As novas tecnologias da informação e comunicação (NTIC) mudaram o curso da história no que tange a força de trabalho, a informação e o conhecimento. Nesse contexto, vários autores buscam identificar como os indivíduos estão sendo inseridos nesse processo. Nessa nova realidade, é inquestionável o potencial de inserção social por meio da inclusão digital.

Apesar de não haver um consenso sobre os resultados da inclusão digital, alguns autores relatam que a inclusão digital consiste de apenas disponibilizar infraestrutura de 
acesso a Internet. Outros destacam que se trata da apropriação do conhecimento pelo uso das NTIC e que a inclusão digital não promove a inserção social do individuo se houver pouca ou nenhuma ação educacional. Mas há consenso de que a inclusão digital não promove necessariamente a inclusão social. O individuo com treinamento em informática não terá necessariamente a garantia de um emprego que mudará sua realidade social. No entanto, o aprendizado do uso da tecnologia da informação pode contribuir para diminuir a pobreza e o analfabetismo.

Nesse sentido, este artigo tem como objetivo avaliar o impacto da qualificação técnica e metodológica de projetos de inclusão social e digital para os indivíduos e o mercado de trabalho no contexto da Java Education and Development Initiative (JEDI) ${ }^{1}$.

O JEDI oferece gratuitamente cursos de Informática via Internet, na modalidade à distância. Seus principais objetivos (i) melhorar a experiência do ensino e da aprendizagem; (ii) aumentar a qualidade dos graduados em Ciência da Computação e Tecnologia da Informação (disponibilizando os materiais de seus cursos gratuitamente para quem se interessar); (iii) aumentar o banco de especialistas em Java; e (iv) fornecer serviços e produtos adequados as necessidades do mercado.

O JEDI nasceu em 2005, na Universidade das Filipinas, apoiado pela Sun Microsystems, através do Java Research \& Development Center (NUNES, 2007). Desde então, o JEDI vem sendo desenvolvido como um projeto colaborativo, contando com o suporte da comunidade Java em todo o mundo. No Brasil, é mantido pelo Brasília Java Users Group (DFJUG) ${ }^{2}$, com o apoio de diversas empresas e instituições.

O DFJUG é uma das 43 comunidades virtuais brasileiras incluídas entre os Java Users Group (JUG). O objetivo desses grupos é disseminar o uso da linguagem de programação de computadores Java por meio de cursos, palestras, listas de discussão, entre outras formas. Esses JUG vêm contribuindo diretamente com o JEDI com ações de tradução de material e aplicação de testes de aprendizado.

Hoje, a tecnologia Java está presente em dois bilhões de dispositivos tecnológicos (OLIVEIRA, 2005). Existia no mundo, já em 2005, cerca de 579 milhões de celulares com Java, 93 operadoras de telefonia móvel, 750 milhões de SmartCards (do tipo JavaCard) distribuídos, 650 milhões de computadores pessoais e 2 milhões de cópias da plataforma de desenvolvimento J2EE. Por isto, 68\% das novas aplicações, hoje, são desenvolvidas em Java, que substituiu o $\mathrm{C}++$ como a competência mais procurada pelos profissionais de desenvolvimento de sistemas.

Segundo Oliveira em meados de 2005, havia uma demanda, em todo o mundo, por dois milhões de novos profissionais de TI a cada ano. Desenvolvedores Java devem prover soluções nas mais diversas áreas de tecnologia. Torna-se crescente a necessidade, por exemplo, do desenvolvimento de soluções para aparelhos celulares integrados com sistemas legados, de maneira a fornecer soluções corporativas. Assim, o conhecimento de Java e de sistemas abertos é imprescindível para profissionais de TI.

O JEDI disponibiliza material de apoio, tais como: manuais, slides de apresentação das aulas, provas, exercícios, material de referência e vídeo-aulas. Conta ainda com softwares, treinamento de instrutores, acesso a suporte e lista de discussão da comunidade. A comunidade JEDI tem contribuído para o sucesso desta iniciativa, que conta no Brasil com mais de 80 voluntários contribuindo ativamente. Os treinamentos são realizados pelos membros do JEDI que conduzem os testes e recebem cada vez mais pessoas como voluntárias. O JEDI possui, atualmente, parcerias globais nas Filipinas, Brasil, Indonésia e Vietnam. Há no mundo, hoje, mais de 200 mil inscritos na Iniciativa JEDI.

\footnotetext{
${ }^{1}$ http://www.dfjug.org.br/DFJUG/jedi/index.jsp

${ }^{2}$ http://www.dfjug.org/DFJUG/jedi/index.jsp
} 
Segundo o boletim informativo DFJUG \#791, de Outubro de 2009, nesta data, a comunidade do JEDI já era formada, no Brasil, por 41.836 participantes.

\section{JEDI como instrumento de Inclusão Social e Digital}

Segundo Scarpa e Melgaço (2006), os modelos de inclusão digital têm um papel central nas questões sociais para promover o bem estar, a geração de renda e o desenvolvimento sustentável, principalmente quando possibilita o diálogo permanente entre organizações sociais, governo, fóruns nacionais, conselhos gestores e de planejamento participativo em ações pró-ativas para a convergência de ações de desenvolvimento. A tarefa não é simples e necessita de processos claros, além de modelos estruturados para avaliação de projetos nessa área. As TIC têm um papel importante no processo de avaliação quando analisada á luz das mudanças sociais desejadas pelos programas públicos e pelas redes sociais informacionais (BRANDÃO, 2009, p. 22).

Firme (2003) relata:

O desafio da avaliação nesse modelo é de reconhecer o valor dessas informações para consolidar entendimentos, apoiar ações necessárias e ampliar o comprometimento e aperfeiçoamento dos indivíduos, grupos, programas, instituições e sistemas enquanto permite a formulação de juízos e recomendações que geram ações, políticas, conhecimento e transformações.

Entre as diversas questões que emergem do exposto é se o conhecimento adquirido em um projeto de inclusão social e digital possibilita de fato a inclusão no mercado de trabalho. Entende-se que avaliar projetos de inclusão digital do ponto de vista social é relevante, uma vez que tal análise pode contribuir não só para os participantes dos cursos do JEDI, mas para qualquer outra iniciativa que porventura apresentem características semelhantes.

As questões formuladas objetivam identificar onde estão esses incluídos, quantos são, se o conhecimento apropriado possibilitou de fato sua inclusão no mercado de trabalho e se as pessoas foram incluídas social e digitalmente.

\section{Aspectos metodológicos}

Entre as propostas metodológicas para a avaliação de projetos de inclusão digital e social estão (Warschauer, 2006), (Rondelli, 2003) e (Sorj, 2003), que defendem uma metodologia centrada na convergência de recursos digitais (linguagem e conteúdo), físicos (computadores e conectividade), humanos (letramento e educação) e sociais (comunidades e instituições). O debate atual ocorre no sentido de discutir essa composição, ou seja, equipamentos, acessos, ferramentas, conteúdos, letramento e educação, comunidades e instituições, para melhor definir um projeto de inclusão digital efetivo. Segundo esses autores o modelo atual adotado por telecentros são ineficientes no processo de inclusão digital. Para esse trabalho, destacam-se o Modelo de Avaliação de Programa de Inclusão Digital e Social - MAPIDS (BRANDÃO, 2009) e a Matriz de Avaliação de Projetos de Inclusão Digital (LEMOS, 2007).

Segundo Brandão (2009, p. 31), seu MAPIDS

[...] é proposto segundo uma abordagem ampliada da avaliação orientada ao participante. Nesse modelo, os princípios políticos e metodológicos são adaptados aos métodos tradicionais de mensuração quantitativa aplicados às análises multidimensionais

e qualitativas. Procurou-se adequar os métodos científicos da 
avaliação para contextos e situações de intervenção comunitária em redes sociais informacionais, com o uso e suporte das TIC. Os gestores e agentes do programa, em geral não especialistas, participam da avaliação do programa como agentes avaliadores não especialistas, formando uma rede de avaliação com suporte das TIC e da internet.

Nesse modelo, os indicadores de insumos são compostos de descritores de infraestrutura física, tecnológica, humana e orçamentárias. Os indicadores de processos são definidos pelos descritores de gestão administrativa, financeira e orçamentária, social e de conhecimento. Os indicadores de resultados são compostos de descritores de acessibilidade, de habilidades para a inclusão digital, de oportunidades econômicas e sociais e de participação democrática. Os indicadores de inclusão social são compostos de descritores de mudanças observadas nos indivíduos, na unidade e no contexto social. Assim, para este trabalho, foram definidas questões sobre o conhecimento anterior e atual, detalhando especificamente o conhecimento em programação de computadores. O comportamento representado nas repostas define padrões de uso das TIC na vida das pessoas inspirada nos conceitos de inclusão digital, alfabetismos digital, funcional e informacional e competência informacional.

Lemos (2007) propôs uma matriz de análise de projetos de inclusão digital que apresenta uma divisão entre inclusão espontânea e inclusão induzida, essa última divida em três categorias: Técnica, Cognitiva e Econômica. Segundo o autor, a imersão é uma inclusão espontânea. A forma induzida é aquela onde existem espaços para o aprendizado de novas habilidades para o manuseio das novas tecnologias da informação, geralmente em projetos sociais (ONGs, telecentros, cibercafés, etc). Com base nisso, definiu-se, neste trabalho, questões relativas à motivação em participar do JEDI, ao conhecimento anterior e atual em programação. Isso identifica o JEDI como uma iniciativa de inclusão as tecnologias eletrônicas de forma induzida cognitiva, uma vez que o participante tem autonomia e independência no uso complexo das NTIC.

As variáveis para análise empírica escolhidas incluem, então, além do perfil dos participantes do JEDI (UF, idade, escolaridade, ocupação, renda familiar, sexo, estado civil e moradia), outras relativas a motivação, expectativa e desenvolvimento profissional. Com isso, pode-se realizar cruzamentos dessas variáveis qualitativas que demonstra os aspectos da iniciativa JEDI.

Para este estudo, foi aplicado um questionário junto à comunidade JEDI entre 04 à 16 de junho de 2009, via Internet. O questionário foi respondido por 1.173 dos 32.608 participantes à época, alcançando um resultado estatístico de $97 \%$ de confiança. Para se identificar os indivíduos que buscam sua inclusão através do JEDI, selecionaram-se os participantes com renda familiar entre 1 e 3 salários mínimos. Com isso, chegou-se a um universo de 428 respondentes.

\section{Resultados alcançados}

Analisando-se o perfil dos 1.173 respondentes, verificou-se que os participantes da iniciativa JEDI são jovens, com média de idade de 26 anos, e 75\% têm idade inferior a 31 anos. Há predomínio de alunos do sexo masculino (91,1\%), com curso superior (70,8\%), solteiros (62,7\%), sem filhos (61,2\%), sem casa própria (52,3\%), empregado (66,7\%), com rendimento familiar mensal inferior a 5 salários mínimos $(63,4 \%)$, vivendo nas regiões Sudeste ou Centro-Oeste (62,6\%), com predomínio das cidades de Brasília e São Paulo (18,4\% e 17,3\%, respectivamente). Vale destacar a abrangência nacional do curso, já que há alunos em todas as unidades da federação.

Analisando por regiões, verifica-se que no Sudeste está a maior parte dos res- 
pondentes considerados. São Paulo, Minas Gerais e Rio de janeiro totalizam 31\% deles (17\%, 8\% e 6\%, respectivamente). Em segundo lugar, está a região Centro-Oeste, com destaque para o Distrito Federal e Goiás, que totalizam 26\% (16\% e 10\%, respectivamente). Em terceiro lugar, a região Nordeste, onde se destacam Ceará e Bahia, que totalizam $12 \%$ do total nacional (6\% cada um). Mesmo em estados com Internet de baixa velocidade, o que dificulta as oportunidades de aprendizagem, o resultado foi positivo. Uma surpresa foi a baixa participação de representantes da Paraíba, um reconhecido centro tecnológico que conta com o parque tecnológico de Campina Grande e o CEFET (Centro Federal de Educação Tecnológica) de João Pessoa.

A análise dos dados relativos aos 428 respondentes com faixa de renda familiar entre um a três salários mínimos, gerou as Tabelas 1 e 2 .

Quanto aos motivos que levaram os interessados a participar do curso, foram propostos três afirmativas: (i) gratuidade, (ii) novas oportunidades no trabalho atual ou mercado e (iii) tornar-se um multiplicador de conhecimento em projetos sociais. Dentre os respondentes, 59\% discordam como a gratuidade sendo o principal motivador, contra, 38\% que concordam como sendo um dos motivadores para participar da iniciativa JEDI. Entretanto, a concordância de 90\% deixa claro que, para esse universo de respondentes, buscar novas oportunidades, independente de onde seja, no trabalho atual ou no mercado, faz parte do espírito de crescimento profissional. Baseado nisso, pode-se deduzir que esse é o principal motivador para quem participa da iniciativa JEDI.

Tabela 1: Perfil sócio-econômico-cultural da amostra analisada

\begin{tabular}{|c|c|}
\hline Variável & $\%$ \\
\hline \multicolumn{2}{|l|}{ Sexo } \\
\hline Feminino .........................50 & 11,7 \\
\hline Masculino ............................378 & 88,3 \\
\hline \multicolumn{2}{|l|}{ Estado Civil } \\
\hline Solteiro .............................. & 73,4 \\
\hline 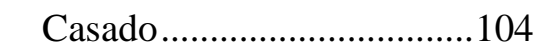 & 24,3 \\
\hline Separado/Divorciado.............10 & 2,3 \\
\hline \multicolumn{2}{|l|}{ Renda familiar } \\
\hline 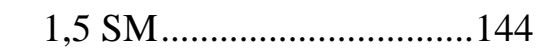 & 33,6 \\
\hline 1,5 a 3 SM & 66,4 \\
\hline \multicolumn{2}{|l|}{ Moradia } \\
\hline Mora com parentes..............136 & 31,8 \\
\hline Alojamento/Hotel/Outros.......8 & 1,9 \\
\hline 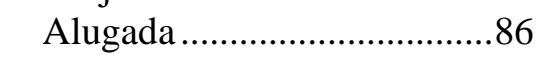 & 20,1 \\
\hline Própria.................................198 & 46,2 \\
\hline
\end{tabular}

\begin{tabular}{|c|c|}
\hline Variável & $\%$ \\
\hline \multicolumn{2}{|l|}{ Ocupação } \\
\hline Desempregado.......................29 & 6,8 \\
\hline Estudante ............................145 & 33,9 \\
\hline Autônomo - Comércio ........... 5 & 1,2 \\
\hline Autônomo - Indústria............. 0 & \\
\hline Autônomo - Serviço.............227 & 6,3 \\
\hline \multicolumn{2}{|c|}{ Empregado Empresa Privada16939,5 } \\
\hline Servidor Público ...................34 & 7,9 \\
\hline Outra ocupação .....................19 & 4,4 \\
\hline \multicolumn{2}{|l|}{ Escolaridade } \\
\hline Fundamental.......................... & 1,6 \\
\hline Médio ................................167 & 39,0 \\
\hline Graduação ..........................2222 & 51,9 \\
\hline Especialização........................30 & 7,0 \\
\hline Mestrado .............................2 & 0,5 \\
\hline
\end{tabular}


Tabela 2 - Resumo das afirmações da pesquisa de campo limitado a 3 Salários Mínimos

Motivação: Por que fez ou está fazendo o curso?

a) Gratuidade

Concordam Discordam

b) Novas oportunidades no trabalho atual ou mercado

(\%)

38,3

(\%)

Tornar-se um multiplicador de conhecimento em projetos

sociais

90,0

59,9

78,2

8,8

Expectativas: Quais são suas expectativas quanto ao curso?

a) Aprender uma nova profissão/Mudar de área de trabalho

b) Tornar-se um especialista em JAVA, inclusive obter uma

b) certificação

c) Melhorar a remuneração

$\begin{array}{lr}48,0 & 46,9 \\ 89,8 & 4,6 \\ 89,3 & 5,8\end{array}$

\section{Desenvolvimento profissional: Qual sua evolução a partir desse} aprendizado?
a) O curso trouxe novas oportunidades

39,4
51,7
b) Você vai aplicar e/ou aplicou o aprendizado no seu trabalho
77,0
11,4
curso
74,5
11,8
d)
e) Você acha que esse aprendizado incentivará a novos cursos trabalho
Sua remuneração melhorou com a aplicação desse conhecimento no
32,9
44,1
no JEDI?
95,4

Analisando os percentuais, 78\% de concordância, verifica-se que quanto menor a renda familiar, maior a importância das pessoas quanto a essa afirmativa. A preocupação com o social está representada como sendo a segunda afirmativa mais importante. Nessa questão, percebe-se que o principal motivador para participar do JEDI é a busca por novas oportunidades de trabalho, totalizando 90\% de concordância. Contudo, apenas receber esse novo conhecimento para uma melhor colocação profissional não é tudo. Esse mesmo participante quer também contribuir socialmente, e isso, está representado por 78\% que querem, sim, novos conhecimentos para ajudar também outras pessoas.

Martini (2005) afirma que o objetivo da Inclusão Digital é o uso livre da tecnologia da informação, com a ampliação da cidadania, o combate à pobreza, a garantia da privacidade e da segurança digital do cidadão, a inserção na sociedade da informação e o fortalecimento do desenvolvimento local. E isso fica claro quando se pergunta Por que fez ou está fazendo o curso? Se é pela gratuidade ou busca de novas oportunidades no trabalho ou, simplesmente, tornar-se um multiplicador de conhecimento em projetos sociais. Pode-se interpretar como uma liberdade de escolha?

Se a principal motivação, representada por mais de $90 \%$ dos respondentes, é a busca por novas oportunidades no trabalho atual e no mercado, qual seria então a expectativa quanto ao aprendizado? O mercado de trabalho na área de tecnologia da informação é a que mais oferece vagas de emprego, mas também a que menos tem pessoal qualificado. E aqui não se está discutindo qualidade, mas sim quantidade. Diante dessa realidade, será que as pessoas estão mesmo buscando aprender uma nova profissão ou mudar de área de trabalho, ou melhor, tornar-se um especialista, inclusive com certificação? Talvez busquem simplesmente remuneração. Para chegar a essa conclusão, analisou-se as respostas do bloco III.

Os percentuais ficam quase iguais quando analisamos a concordância e a discor- 
dância (48\% e 46\%, respectivamente) quanto à afirmativa que estão buscando aprender uma nova profissão / mudar de área de trabalho. Se não existe praticamente diferença, então não é esse o objetivo desse grupo.

Atualmente, o mercado está valorizando muito especialistas com certificação em qualquer área de TI. Em desenvolvimento não poderia ser diferente. Uma análise simples de mercado evidencia que a posse de uma certificação acarreta em melhores salários. E isso faz diferença, não só financeira, mas também técnica. Por isso, os números refletem essa realidade, pois há $89 \%$ de concordância quanto a essa afirmativa.

Apesar da importância em se ter uma certificação, deve haver contrapartida do ponto de vista de remuneração. 89\% dos respondentes concordam com isso.

Quanto à evolução do conhecimento, será que, conforme indagação de Paulo Freire, “houve transformação?”. Verificou-se claramente que o conhecimento adquirido trouxe novas oportunidades profissionais e salariais, inclusive no trabalho. Nesse mesmo contexto de evolução e satisfação, questiona-se se esse aprendizado incentivará a novos cursos no JEDI.

Avaliando o resultado da primeira afirmativa, o fato de que os respondentes ainda estão realizando os cursos do JEDI, parece ter contribuído para as respostas de 51\% da amostra. Tal avaliação poderá indicar resultados mais consubstanciosos após o término do curso, uma vez que entre o processo de aprendizado e a implementação demanda tempo para reconhecimento social e profissional, associada, inclusive, à mudança de equipes de trabalho interna e externa à organização de trabalho atual. Além disso, como ainda não são especialistas certificados, as oportunidades de trabalho de maior reconhecimento técnico e salarial não foram concretizadas. No entanto, o JEDI trouxe novas oportunidades para 39\% dos respondentes. Assim, nesse grupo, houve sim, transformação, isso pode estar relacionado à inserção no mercado de trabalho ou uma promoção no trabalho atual. Então, o curso já representa alterações para o individuo, o que permite concluir que o JEDI oportuniza às pessoas a mudarem sua realidade social.

Mesmo em discordância sobre novas oportunidades, 76\% vão aplicar ou aplicou o aprendizado no trabalho. Como essa afirmativa é aberta, isto é, irá aplicar ou já aplicou, A resposta pode significar que esse aprendizado vai ser aplicado futuramente no trabalho, dependendo apenas de novas oportunidades ou o individuo já trabalha com JAVA e aplica o conhecimento no seu dia-a-dia.

Entre os respondentes, $74 \%$ evoluíram profissionalmente com o novo conhecimento. È um grande resultado, pois, contribui para responder se o curso trouxe oportunidades quanto ao crescimento profissional. Apesar dos 51\% de discordância, 39\% relatam crescimento profissional, corroborando os aspectos positivos da inserção no JEDI.

Apesar dessa evolução profissional, 44\% não tiveram melhorias quanto à remuneração pela aplicação desse novo conhecimento no trabalho. Como o curso ainda está em andamento, os respondentes ainda estão exercendo suas antigas tarefas, o que contribui para a permanência de salários equivalentes a três salários-mínimos. Contudo, para 33\% do universo pesquisado, a remuneração já apresenta melhoras. Isso demonstra que o aprendizado no JEDI contribui para alterações na realidade financeira.

Analisando do ponto de vista de inclusão digital e social, o curso trouxe novas oportunidades para 39\% dos respondentes, melhorou a remuneração de 33\% deles. Esses números reforçam a importância da iniciativa. A satisfação é confirmada pelos $80 \%$ de respondentes que tencionam participar de novos cursos no JEDI.

\section{Considerações finais}

Um questionário foi então construído a partir dos modelos de Brandão (2009) e Lemos (2007), objetivando mapear o perfil, a motivação, a expectativa e o desenvolvi- 
mento profissional dos participantes do JEDI. A objetivo era identificar se houve mudança na realidade social do individuo participante a partir do conhecimento adquirido: foi possível entrar no mercado de trabalho? Melhorar sua condição financeira? Esse conhecimento trouxe novas oportunidades?

Os resultados dessa pesquisa demonstram que os participantes do JEDI têm as habilidades de uso das TIC, apresentado no Indicador de Alfabetização Digital e Social (IADS) para mensuração do grau de alfabetização digital e social dos indivíduos da iniciativa.

Os resultados apresentam um conjunto de informações que mostram que a iniciativa JEDI não é restrita a um meio para se iniciar nos conhecimentos inerentes à Tecnologia da Informação. Trata-se também de aprimoramento de conhecimento em desenvolvimento de sistemas de informação. Os números apresentados mostram que entre os participantes da iniciativa JEDI com renda familiar entre 1 e 3 salários mínimos, 83\% tinha conhecimento anterior em programação de computadores, 58\% tinha conhecimento em programação orientada a objeto e $63 \%$ já trabalhava como desenvolvedor. Além disso, 52\% são graduados e 7\% possuem pós-graduação.

É importante ressaltar que $16 \%$ dos respondentes não tinha conhecimento anterior em programação e $11 \%$ não tinha conhecimento de lógica de programação. Avaliando o conhecimento atual, $41 \%$ não têm conhecimento de programação orientada a objeto e apenas 36\% não trabalha como desenvolvedor. Diante desse fato, sugere-se a realização de estudos futuros para saber quantos desses iniciantes se transformaram em novos desenvolvedores.

De acordo com Paulo Freire, “educar é transformar as pessoas”, logo esta pesquisa, prioritariamente, optou pelo aprofundamento da compreensão se uma iniciativa como o JEDI contribui para isso. Em síntese, os resultados demonstraram que o JEDI está cumprindo esse papel.

\section{Referências}

BRANDÃO, M. F. R. Um Modelo de Avaliação de Projeto de Inclusão Digital e Social. Tese (Doutorado em Psicologia Social, do Trabalho e das Organizações), UnB, Brasília, 2009.

FIRME, T. P. Avaliação em rede. Relatório Técnico. Rio de Janeiro: Rede de Informações para o Terceiro Setor - RITS, 2003.

LEMOS, A. (Org). Cidade digital: portais, inclusão e redes no Brasil. Salvador, BA: EDUFBA, 2007. 206 p.

MARTINI, R. Inclusão digital \& inclusão social, Revista Inclusão Social, Vol. 1, $\mathrm{N}^{\mathrm{o}} 1$, 2005. Disponível em <http://revista.ibict.br/inclusao/index.php/ inclusao/article/view/7/13>. Acesso em 21/10/2008.

NUNES, R. JEDI e o ensino de Java gratuito. Revista Mundo Java, nº 20, p. 72, 2006.

OLIVEIRA Jr, D. A. Comunidades de Prática: Um estudo dos grupos de usuários JAVA Brasileiros. Dissertação de Mestrado em Gestão do Conhecimento e Tecnologia da Informação, Universidade Católica de Brasília, 2005.

RONDELLI, E. Quatro Passos para a Inclusão Digital. Revista I-coletiva. 2003. Disponível em: < http://www.comunicacao.pro.br/setepontos/5/4passos.htm>. 
SCARPA, E. V., MELGAÇO, M. A experiência de implantação do balanced scorecard - BSC Vapt Vupt. Palestra apresentada no $12^{\circ}$ Congresso de Informática Pública CONIP. São Paulo, 2006.

SORJ, B. Brasil@povo.com.: a luta contra a desigualdade na sociedade da informação. Rio de Janeiro: Jorge Zahar, 2003.

WARSCHAUER, M. Tecnologia e inclusão social: a exclusão digital em debate. São Paulo: Senac, 2006. 